easy to deal with from the dynamical point of view. The quasi- (but not exact) coincidence could be a result of the approximation, both theoretical and experimental, or perhaps an essential feature of the phenomenon.

Osservatorio Astrofisico di Arcetri,

G. Calamai Firenze, Italy.

${ }^{1}$ Fan, C. Y., Mayer, P., and Simpson, J. A., Space Research, 2 (Amsterdam,

¿Alfvén, H., Cosmical Electrodynamics (Oxford). Calamai, G., Il Nuovo Cimento, X, 26, 1090 (1962).

\section{Polarization Effects on the Profile of the $H \beta$ Line in the Light from the Star $\gamma$ Ursa Majoris}

DURING February 1965 we observed some absorption lines in the spectra of several stars in orthogonal planes of polarization. The measurements were made with a photoelectric grating spectrometer ${ }^{1}$ to which a rotatable 'Polaroid' had been added after the exit slit. The instrument was used at both the Newtonian and Cassegrain foci of the 48-in. telescope of the University of Padua's Observatory at Asiago, Italy.

Differential polarization effects across the absorption lines were sought by looking for departures from the instrumental polarization curve as the spectrometer was scanned.

Öhman ${ }^{2}$ has shown that, in the case of rapidly rotating early-type stars, variations of polarization might appear across the absorption lines, and that, for a simple model, the degree of polarization might attain 0.8 per cent at the centre of the profile.

Considerations of the photometric accuracy required to measure an effect of this size make photographic spectrometry unlikoly to succeed. However, calculations showed that polarizations of this order might just be detected by the instrument mentioned here, using a 1-10-sec integration time, provided the stars were brighter than 6th magnitude.

Observations of five stars were made at the Newtonian focus, the Cassegrain not being normally available for auxiliary instruments. As a result of a preliminary analysis, it seemed that $\gamma(A 0 n e, 2 \cdot \mathrm{m} 5)$ and $\eta(B 3,1 \cdot \mathrm{m} 9)$ Ursa Majoris were worthy of further investigation. We were extremely fortunate in having the Cassegrain focus made available to us and decided to observe $\gamma$ Ursa Majoris, it being the more suitably placed star at the timo of observation.

Of the ten 1- $\AA$ resolution scans taken of the $H \beta$ line of this star, six were taken with the 'Polaroid' transmission axis perpendicular to the exit slit and four with it parallel to the slit. Ratios were formed of the corresponding ordinates of these two sets. The smooth change of this ratio with wave-length represents the variation of the overall instrumental polarization. However, in the neighbourhood of the $\mathrm{H} \beta$ absorption, there are marked deviations from this smooth curve. It can be shown that if $R(\lambda)$ represents the ratio of corresponding ordinates from orthogonal scans at wave-length $\lambda$, and $R_{u}(\lambda)$ the ratio which it is assumed would have been obtained in the absence of source polarization (that is, interpolated values from the smooth curve), then:

$$
P(\lambda)=\frac{R(\lambda)-R_{u}(\lambda)}{R(\lambda)+R_{u}(\lambda)}
$$

where $P(\lambda)=p(\lambda) \cdot \cos 2 \varphi(\lambda), p(\lambda)$ being the degree of polarization and $\varphi(\lambda)$ the angle formed by the direction of the polarized component of the light from the source and the transmission axis of the 'Polaroid'. The value of $p(\lambda)$ can only be separated from $P(\lambda)$ if the absolute instrumental polarization is known. Values of $P(\lambda)$ were evaluated at 1.5- $\AA$ intervals across the line. Both the $H \beta$ profile and $P(\lambda)$ are shown in Fig. 1. The values of

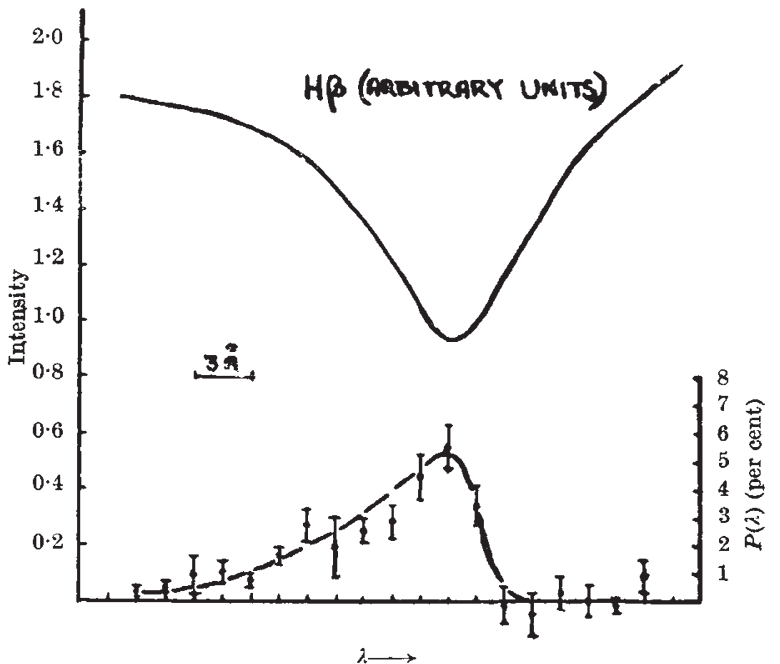

Fig. 1

$P(\lambda)$ are the means of the data and corresponding standard deviations are shown by the bars.

We conclude that:

(1) A differential polarization effect has been observed in the region of the $\mathrm{H} \beta$ line in the light from $\gamma$ Ursa Majoris. Since $p(\lambda) \geqslant P(\lambda)$, the differential polarization across the line must be at least 5 per cent. The magnitude of the effect is surprisingly large if the process suggested by Öhman is occurring. A variation of polarization of this magnitude should just be detectable by photographic spectrometry and, in this connexion, it is interesting to note that Oohman ${ }^{3}$ found 'faint' polarization effects in the $\mathrm{H} \gamma$ line of $\beta$ Lyrae.

(2) The angle $\varphi(\lambda)$ must have been different from $\pi / 4$ since the effect was observed. If $\varphi(\lambda)$ is independent of $\lambda$ over this region, then the form of $p(\lambda)$ and the lino profile exhibit the same asymmetry.

We thank Prof. J. Ring for facilities in his department and Prof. L. Rosino and the staff of the University of Padua's Observatory for their co-operation. This work was supported by the Department of Scientific and Industrial Research.

D. Clarke

J. F. Grainger

Department of Applied Physics, University of Hull.

${ }^{1}$ An improved version of the instrument referred to in: Grainger, J. F., and Ring, J., Mon. Not. Roy. Astro. Soc., 125, 93 (1963).

2 Öhman, Y., Astrophys. J., 104, 460 (1946).

${ }^{3}$ Öhman, Y., Nature, 134, 534 (1934).

\section{PHYSICS}

\section{Narrow Spectral Emission from a Passively Q-spoiled Neodymium-glass Laser}

THE spectral behaviour of the Nd-glass laser has recently attracted much attention. Normally the output of the Nd-glass laser consists of a broad band of wavelengths (approximately $50 \AA$ wide) composed of hundreds of lines centred about $1 \cdot 06 \mu$ (see Fig. 1 $A$ ). This structure has been described in detail by Snitzer ${ }^{1}$. Time-resolved spectra, 1,2 and investigations of the pumping energy dependence of the output band-width ${ }^{3}$ all may be interpreted on the basis of inhomogeneous behaviour of the $\mathrm{Nd}^{+3}$ emission. The purpose of this communication is to report the efficient generation of a single narrow spectral line from a giant pulsed $\mathrm{Nd}^{+3}$ glass laser by passivo $Q$-spoiling. 